\title{
Measurement of Complex Acoustic Intensity in an Acoustic Waveguide
}

\author{
Wenbo Duan \\ School of Engineering and Design, Brunel University, \\ Uxbridge, Middlesex, UK, UB8 3PH \\ Ray Kirby \\ School of Engineering and Design, Brunel University, \\ Uxbridge, Middlesex, UK, UB8 3PH \\ e-mail: ray.kirby@brunel.ac.uk
}

Jevgenija Prisutova

School of Engineering, University of Bradford,

Bradford, UK, BD7 1DP

Kirill V. Horoshenkov

School of Engineering, University of Bradford,

Bradford, UK, BD7 1DP 


\begin{abstract}
Acoustic intensity is normally treated as a real quantity, but in recent years many articles have appeared in which intensity is treated as a complex quantity where the real (active) part is related to local mean energy flow, and the imaginary (reactive) part to local oscillatory transport of energy. This offers the potential to recover additional information about a sound field and then to relate this to the properties of the sound source and the environment that surrounds it. However, this approach is applicable only to a multi-modal sound fields, which places significant demands on the accuracy of the intensity measurements. Accordingly, this article investigates the accuracy of complex intensity measurements obtained using a tri-axial Microflown intensity probe by comparing measurement and prediction for sound propagation in an open flanged pipe. Under plane wave conditions comparison between prediction and experiment reveals good agreement, but when a higher order mode is present the reactive intensity field becomes complicated and agreement is less successful. It is concluded that the potential application of complex intensity as a diagnostic tool is limited by difficulties in measuring reactive intensity in complex sound fields when using current state of the art acoustic instrumentation.
\end{abstract}




\section{INTRODUCTION}

Sound intensity has long been used to describe the flow of energy in sound fields.

Conventional practice is to take the time average of the instantaneous sound intensity and to treat intensity as a real quantity ${ }^{1}$. Thus, when the acoustic particle velocity is in phase with the pressure one fully captures the characteristics of the energy flow in the sound field, for example when a plane wave propagates in a free field. However, when the particle velocity and pressure are not in phase additional information may be obtained by accounting for this phase difference, and this can be realised by treating intensity as a complex quantity where the real part represents the magnitude of the local mean energy flow, and the imaginary part the local oscillatory transport of energy ${ }^{1}$. Accordingly, complex intensity will deliver additional information regarding energy transport but only in regions of sound scattering and/or when higher order modes are propagating in a waveguide. It is attractive to try and make use of this additional information when studying, for example, the near field of a sound source and to use this to help identify relevant characteristics of the source. Here, a number of studies have appeared in the literature which seek to do just this ${ }^{2-5}$, with some limited qualitative success being observed following measurements of radiated sound fields. A question mark remains, however, over the accuracy that may be achieved using this type of approach and hence the general viability of complex intensity when used as a diagnostic tool. This article seeks to quantify the levels of accuracy that one may achieve for complex intensity measurements under controlled laboratory conditions for which the measured complex intensity is expected to compare well with theoretical predictions. This then permits a direct quantitative investigation into the feasibility of using a 3-component complex intensity vector as a condition diagnostic tool in multi-modal waveguides. 
In general, sound intensity is normally referred to in terms of the instantaneous intensity, although Jacobsen ${ }^{6}$ noted that for a non-monochromatic sound field one cannot "make an instantaneous separation of the particle velocity into components in phase and quadrature with pressure". This means that for a non-monochromatic sound field multiple definitions for instantaneous complex intensity are possible. Jacobsen ${ }^{6}$ reviews a number of alternative definitions and concludes that the one suggested by Heyser ${ }^{7}$ is probably the most useful. Here, Heyser ${ }^{7}$ uses Hilbert transforms to decompose the instantaneous intensity into real and imaginary components, which are shown by Jacobsen ${ }^{6}$ to be generalisations of the instantaneous complex intensity used for monochromatic sound fields. A series of articles later introduce an alternative definition for instantaneous intensity in a general nonmonochromatic sound field ${ }^{8-10}$. In particular, Stanzial and Prodi ${ }^{10}$ called these new parameters the radiating and oscillating intensities, which provide an alternative to the more usual active and reactive instantaneous intensity terminology. These definitions were chosen so that the time average of the radiating intensity equals the time average of the instantaneous intensity, and the time average of the oscillating intensity is zero. Stanzial et al. ${ }^{11}$ also reinterpret the acoustic energy flow using an electrical analogy in order to define an acoustic power factor as the ratio of the active intensity to the complex intensity magnitude. The definition of complex intensity suggested by Stanzial and Prodi ${ }^{10}$ is significantly different to that proposed by Heyser ${ }^{7}$, and so in this article the relative merits of each approach will be investigated. However, what is of most interest here is the use of Stanzial and Prodi's complex intensity definition in the study of sound radiation from complex noise sources. This presents the possibility of using complex intensity to aid in the deduction of relevant characteristics of a sound source by providing additional acoustical information which may be used to improve inverse analysis procedures. This approach has the potential for use in a wide range of applications and the authors are currently interested in using complex intensity 
as an inverse analysis tool for the investigation of the characteristics of obstructions in a multi-modal waveguide.

The use of complex intensity in inverse analysis has so far been restricted to sound radiation problems. For example Stanzial and Prodi ${ }^{10}$ measured instantaneous complex intensity patterns radiated by a point source located in an anechoic chamber, and also in a reverberation room where the authors note some difficulties when attempting to link their new definitions to more usual interpretations. Measurements were later extended to more complex sound fields by Mann and Tichy ${ }^{2}$ who measured instantaneous intensity in the acoustic near field of resonant cavities and vibrating plates. Here, Mann and Tichy propose that one may deduce the vibrational characteristics of a plate using reactive intensity, although they note that some prior knowledge of the physical characteristics of the sound source is necessary. Mann and Tichy propose that instantaneous reactive intensity is especially useful in analyzing the acoustic near field and can be used for machine diagnostics, although they note that reactive intensity can only provide a qualitative guide when attempting to distinguish between different noise sources, since the instantaneous reactive intensity field is not necessarily unique, see also Mann and Tichy ${ }^{12}$. Other applications include the measurement of complex intensity fields radiated by a moped ${ }^{4}$, and the instantaneous intensity field scattered by a rigid prolate spheroid ${ }^{13}$. Barton et al. ${ }^{5}$ later investigated the structural features of the scattered wave field in the resonance region of a motionless rigid sphere, where instantaneous complex intensity was shown to reveal the interference patterns of the diffracted waves. Barton et al. ${ }^{14}$ also extended this analysis by considering a sphere that could either be fluid-filled or respond like an elastic thin-walled shell. Jacobsen and Molares ${ }^{15}$ studied the fundamental statistical properties of the instantaneous active and reactive sound intensity in reverberant spaces at low frequencies and 
demonstrated the influence of modal overlap on the relative variance in the instantaneous active and reactive intensity fields. Dean and Braselton ${ }^{16}$ also showed that this approach can be applied to study the instantaneous intensity vector flow in compressional waves in elastic media.

Thus, instantaneous complex intensity has been used in a number of sound radiation applications and its potential use as a diagnostic tool has been investigated by a number of authors. For sound radiation problems this approach does, however, require the measurement of complex intensity in the acoustic near field of a sound source. A crucial assumption of this approach is that one is able to measure complex intensity accurately enough to enable its use in any inverse analysis procedure that follows. However, it is very difficult to quantify the accuracy of this type of approach for external sound radiation problems; this is because developing accurate theoretical models suitable for comparison against the measured data is challenging. Accordingly, this article compares instantaneous and non instantaneous complex intensity measurements against predictions for sound propagation inside a circular duct, as this presents a much more controlled environment under which to review the accuracy of complex intensity measurements. Moreover, if one also studies a stationary sound field then the reactive intensity is likely to be significant over the entire length of the duct because both incident and reflected waves will propagate, so that one is not restricted solely to measuring close to a region of high modal scattering. Accordingly, this article begins in Section II by reviewing two methods of representing instantaneous complex intensity measurements, the methods of Heyser ${ }^{7}$ and Stanzial and Prodi ${ }^{10}$. Here, use is made of a simple duct acoustics model in order to demonstrate some of the advantages and disadvantages of each method for the measurement of instantaneous intensity. The two methods are then compared against one another in Section IV through the prediction and 
measurement of sound scattered from the open end of a flanged duct. Here, both instantaneous and non instantaneous intensity is investigated in order to provide further opportunities to make quantitative comparisons between prediction and experiment. Experimental data is obtained using a tri-axial "p-u" Microflown sensor ${ }^{17,}{ }^{18}$, which combines a pressure microphone with three particle velocity transducers to measure simultaneously the air particle velocity in three orthogonal directions. The advantage of using an open ended flanged duct is that it enables the accurate placing of the Microflown sensor, as well as permitting the study of sound scattering in a controlled environment. This does, however, require a more sophisticated theoretical model capable of capturing sound scattering close to the duct exit, as well as the propagation of higher order modes in the duct, and this [numerical] model is briefly described in section III.

\section{COMPLEX INTENSITY DEFINITIONS}

Jacobsen $^{6}$ suggests that when measuring the instantaneous complex sound intensity in a nonmonochromatic sound field it is preferable to use a narrow band source in order to apply the complex representation of the instantaneous intensity suggested by Heyser ${ }^{7}$. In this respect, the study of instantaneous complex intensity in a non-monochromatic sound field is challenging and so if one is attempting to compare prediction and experiment it is sensible first to simplify the problem and use only a monochromatic sound field. For a monochromatic sound field, the real part is normally called the instantaneous active intensity and the imaginary part the instantaneous reactive intensity ${ }^{1,9}$. However, it is common also to measure intensity in a stationary sound field, and this is normally accomplished by taking a time average of the instantaneous intensity and treating intensity as a real quantity. Here, the 
real part is often called the active or mean active intensity ${ }^{1}$ so that it corresponds to the terminology used for the instantaneous active intensity discussed previously. This terminology appears to have been chosen in view of the ubiquity of the real part of the time averaged intensity, although it is noted here that other authors sometimes refer to this quantity as the time independent intensity. This definition works well for the real part of the instantaneous complex intensity, although if one simply takes the time average of the imaginary part of the instantaneous intensity then this will give zero. To address this, Fahy ${ }^{1}$ uses the amplitude of the imaginary part of the instantaneous intensity and defines this as the reactive intensity. Thus, for instantaneous complex intensity, Fahy adopts the terminology of instantaneous active and instantaneous reactive intensity, and for non instantaneous intensity, active and reactive intensity. In view of the simplicity of this approach, the terminology of Fahy is also adopted here.

Two techniques for calculating of the instantaneous complex intensity are investigated here. The first technique is based on the method proposed by Heyser ${ }^{7}$, who defines the instantaneous active $\mathbf{I}(t)$ and reactive $\mathbf{J}(t)$ intensity as

$$
\mathbf{I}(t)=\frac{1}{2} p \mathbf{u}+\frac{1}{2} \hat{p} \widehat{\mathbf{u}}
$$

and

$$
\mathbf{J}(t)=\frac{1}{2} \hat{p} \mathbf{u}-\frac{1}{2} p \widehat{\mathbf{u}},
$$

respectively. Here, $t$ is time, and $p$ and $\mathbf{u}$ are the time history values of pressure and velocity, respectively, with ${ }^{\wedge}$ denoting a Hilbert transform. The second technique was introduced by Schiffrer and Stanzial ${ }^{8}$, Stanzial et al. ${ }^{9}$, and Stanzial and Prodi ${ }^{10}$ who define radiating and oscillating components of instantaneous intensity so that 


$$
\mathbf{I}_{\mathrm{rad}}(t)=\frac{p^{2}\langle p \mathbf{u}\rangle}{\left\langle p^{2}\right\rangle}
$$

and

$$
\mathbf{J}_{\mathrm{osc}}(t)=\frac{\left\langle p^{2}\right\rangle p \mathbf{u}-p^{2}\langle p \mathbf{u}\rangle}{\left\langle p^{2}\right\rangle}
$$

Here $\langle\cdot\rangle$ indicates a time average, and $\mathbf{I}_{\mathrm{rad}}(t)$ and $\mathbf{J}_{\mathrm{osc}}(t)$ are the radiating and oscillating intensity, respectively. It is interesting to note here that the method of Stanzial and Prodi ${ }^{10}$ introduces a time averaging procedure that does not appear in the method of Heyser ${ }^{7}$. For time stationary problems this presents no problems, however it is clear that for transient problems the calculation of complex intensity will depend on the time window chosen for the averaging process. Here, Stanzial and Prodi ${ }^{10}$ propose that their method may be applied to transient problems provided one restricts the analysis to narrow banded signals.

The relative performance of both methods for characterising instantaneous intensity may first be investigated by using a simple duct acoustics model in which a simple obstruction/area discontinuity is placed within a duct. This will enable the study of two different signals, one incident in the duct and one reflected by the obstruction. Here, the details of the obstruction are not important, rather it is the way in which the two signals are considered in the time averaging process in Eqs. (3) and (4) that is of interest. Therefore, consider a circular duct of diameter $d$, in which an obstruction of arbitrary shape is placed at an axial distance of $x=L$, with a narrow band incident pulse generated by a sound source placed at $x=0$. If the analysis is restricted to plane wave propagation then the acoustic pressure $p \propto e^{-i(k x-\omega t)}$, where $t$ is time, $i=\sqrt{-1}$, and $k=\omega / c$, with $\omega$ the radian frequency and $c$ the speed of sound. The complex intensity in the duct may then be calculated at any given location in the duct using the following equations ${ }^{1}$, 


$$
\mathbf{I}(t)=0.5 \operatorname{Re}\left\{p \mathbf{u}^{*}\right\}[1+\cos 2(\omega t+\emptyset)]
$$

and

$$
\mathbf{J}(t)=0.5 \operatorname{Im}\left\{p \mathbf{u}^{*}\right\} \sin 2(\omega t+\emptyset)
$$

where, $\mathbf{u}^{*}$ is the complex conjugate of the velocity vector $\mathbf{u}$, and $\emptyset$ is the phase of the pressure. For plane wave propagation, one may readily separate the axial intensity $\mathrm{I}_{x}(t)$ and $\mathrm{J}_{x}(t)$ computed for the incident pulse from that of the pulse reflected from the obstruction provided the location of the obstruction is sufficiently far from the point at which the intensities are computed (in order to separate the two signals in the time domain). In Fig. 1, predictions obtained using the methods of Heyser ${ }^{7}$ and Stanzial and Prodi ${ }^{10}$ are compared to one another for the instantaneous active intensity in Fig 1a, and instantaneous reactive intensity in Fig. 1b. In these figures only the incident and reflected signals are presented and, when computing the time average in Stanzial and Prodi's definition, the upper time limit is taken so as to encompass both the incident and reflected pulse.

It is immediately apparent in Fig. 1 that the methods of Heyser ${ }^{7}$ and Stanzial and Prodi ${ }^{10}$ deliver very different representations of complex intensity. For the active intensity the method of Heyser delivers the upper envelope of $\mathrm{I}_{\operatorname{rad}_{x}}$ for the incident and reflected sound wave, although it is noticeable that the sign is reversed for the reflected wave. For the reactive/oscillating intensity Heyser's method gives a value of zero, whereas Stanzial and Prodi predict oscillating energy is present in the duct. This latter result illustrates the 
problems associated with taking the time average in Eqs. (3) and (4). This simple problem has been chosen because it is easy to show that for plane wave propagation using a transient signal there can be no oscillating energy in the duct. Thus, the method of Heyser delivers exactly what one would expect to see (a zero imaginary part), but if one takes both pulses when carrying out the time average then the method of Stanzial and Prodi delivers incorrect values, as the imaginary component is not zero. It is clear, therefore, that one must be very careful when implementing the time averaging process in Eqs. (3) and (4), and it is seen in Fig. 1 that cancelling can occur between signals and for this example this delivers erroneous results. This problem may be rectified by appropriate windowing, so that the incident and reflected waves are analysed separately before computing $\mathrm{I}_{\operatorname{rad}_{x}}(t)$ and $\mathrm{J}_{\mathrm{osc}_{x}}(t)$. If this is carried out then the method of Stanzial and Prodi ${ }^{10}$ delivers consistent predictions for the real and imaginary parts of the intensity, and the method of Heyser is seen to provide a running time average of $\mathrm{I}_{\operatorname{rad}_{x}}(t)$ and an envelope of $\mathrm{J}_{\mathrm{osc}_{x}}(t)$. But this is only possible if one can successfully window out each pulse.

The predictions in Figs. 1a and 1b demonstrate that one must be careful when using the method of Stanzial and Prodi ${ }^{10}$ when analysing transient problems. If multiple signals are included in the time averaging procedure (and these need not overlap one another) then interference may occur and distorted values of complex intensity may arise. This type of behaviour may explain some of the problems identified when complex intensity measurements were undertaken in a reverberation room by Stanzial and Prodi ${ }^{10}$. Furthermore, when studying transient signals radiated by a sound source it is possible that different noise sources may interfere with one another, and after carrying out a time averaging process oscillating energy may be predicted when it is not actually present in 
practice. Accordingly, one needs to exercise caution when applying the method of Stanzial and Prodi ${ }^{10}$, especially in the study of the radiation of sound from complex noise sources, including, for example those noise sources studied in a later article ${ }^{4}$.

The definitions of Heyser ${ }^{7}$ and Stanzial and $\operatorname{Prodi}^{10}$ are principally of use in the study of instantaneous intensity. For a time stationary monochromatic sound field it is still possible to use these definitions by taking a time average of the instantaneous intensity; however, for non instantaneous intensity the definition of complex intensity is unambiguous and here it is usual practice to take a cross-spectrum between the measured sound pressure and particle velocity in order to compute the frequency dependent active and reactive intensity ${ }^{1,19}$. Thus, for noninstantaneous intensity

$$
\mathbf{I}(\omega)=\operatorname{Re}\left[\mathrm{S}_{p \mathbf{u}}(\omega)\right]
$$

and

$$
\mathbf{J}(\omega)=-\operatorname{Im}\left[\mathrm{S}_{p \mathbf{u}}(\omega)\right]
$$

Here, $\mathbf{I}(\omega)$ is the active and $\mathbf{J}(\omega)$ the reactive intensity in a time stationary sound field, where $S_{p \mathbf{u}}(\omega)$ denotes the cross spectrum for $\omega \geq 0$, and the minus sign in Eq. (8) appears because the reactive intensity is defined as pointing in the direction of decreasing pressure. Here, the study of time stationary problems is attractive as this simplifies the measurements and the definition of complex intensity is also unambiguous. However, the measurement of complex intensity in a time stationary problem is not necessarily straightforward because if one wishes to compare measurements against predictions one needs to ensure that the respective boundary conditions of the problem are well known so that they can be accurately represented in the theoretical model. In view of this it was decided here to measure intensity 
in an open ended duct as this would make it easy to locate the intensity probes close to a region of modal scattering, as well as delivering a well defined problem in terms of the termination of the duct. The disadvantage of this experimental set up is that it requires a more advanced theoretical model so this is discussed next, before moving on to compare predictions against experiment. Here, the theoretical prediction of non instantaneous intensity may be obtained from the following equations

$$
\mathbf{I}(\omega)=0.5 \operatorname{Re}\left\{p \mathbf{u}^{*}\right\}
$$

and

$$
\mathbf{J}(\omega)=0.5 \operatorname{Im}\left\{p \mathbf{u}^{*}\right\}
$$

where $\mathbf{I}_{q}(\omega)$ is the active and $\mathbf{J}_{q}(\omega)$ is the reactive intensity. These values are then computed using the theoretical model that follows.

\section{THEORY FOR A FLANGED DUCT}

The prediction of sound radiated by a flanged duct is far from straightforward, especially if one wishes to include evanescent modes scattered by the end of the duct, as well as higher order modes propagating modes in the duct itself. Recently, a suitable numerical model was proposed by Duan and $\mathrm{Kirby}^{20}$ and this model is adapted here to include a point source at the closed end of the duct, see Fig. 2. The model adopts modal expansions for the uniform region $\mathrm{R}_{2}$, and the outer region $\mathrm{R}_{4}$; a finite element discretisation is used to capture the sound field radiated by a point source in region $R_{1}$, and in region $R_{3}$ a relatively dense mesh is used 
to capture the complex scattered sound field from the end of the duct. The details of the finite element based model are described by Duan and Kirby, and so only a brief summary is provided here. The open end of the duct is assumed to have an infinite flange, which in practice means that the length of the flange is much larger than the wavelength of sound considered. Sound propagation in region $R_{q}(q=1,2,3$ and 4$)$ is governed by the following acoustic wave equation

$$
\frac{1}{c^{2}} \frac{\partial^{2} p_{q}}{\partial t^{2}}-\nabla^{2} p_{q}=F_{1}
$$

where $F_{1}$ is assumed to be a time harmonic sound source in region $\mathrm{R}_{1}$, and is given as

$$
F_{1}=\delta\left(r-r_{0}\right) \delta\left(\theta-\theta_{0}\right) \delta\left(z-z_{0}\right) e^{i \omega t}
$$

for a cylindrical coordinate system $(r, \theta, z)$, with $\left(r_{0}, \theta_{0}, z_{0}\right)$ denoting the location of the monopole sound source. A finite element discretisation is used so that the pressure $p=\mathbf{N p}$, where $\mathbf{N}$ and $\mathbf{p}$ are row and column vectors, respectively, and these hold the global trial (or shape) functions and the unknown acoustic pressures. After applying the Galerkin method, the governing equation in region $R_{1}$ can be written as

$$
\int_{\Omega_{1}}\left[\nabla \mathbf{N}_{\mathbf{1}}^{T} \nabla \mathbf{N}_{\mathbf{1}}-k_{1}^{2} \mathbf{N}_{\mathbf{1}}^{T} \mathbf{N}_{\mathbf{1}}\right] d \Omega_{1} \mathbf{p}_{\mathbf{1}}=\int_{\Gamma_{\mathrm{A}}} \mathbf{N}_{\mathbf{1}}^{T} \nabla p_{1} \cdot \mathbf{n}_{A} d \Gamma_{1}+\int_{\Omega_{1}} \mathbf{N}_{\mathbf{1}}^{T} F_{1} d \Omega_{1}
$$

with

$$
\int_{\Omega_{1}} \mathbf{N}_{\mathbf{1}}^{T} F_{1} d \Omega_{1}=\mathbf{N}_{\mathbf{1}}^{T}\left(r_{0}, \theta_{0}, z_{0}\right)
$$

Here, $\Omega_{1}$ denotes the volume of region $R_{1}$, and $\mathbf{n}_{\boldsymbol{A}}$ is the outward unit normal vector over surface $\Gamma_{\mathrm{A}}$; the surfaces of region $R_{1}$ that do not lie on $\Gamma_{\mathrm{A}}$ are assumed to be hard walled so 
the integral over these surfaces in Eq. (13) is zero. The finite element discretisation in region $R_{1}$ is joined to the rest of the problem using the hybrid finite element method described by Duan and Kirby ${ }^{20}$. This requires the pressure in regions $R_{2}$ and $R_{4}$ to be expanded over a series of eigenmodes to give

$$
p_{2}(r, \theta, z)=\sum_{n=0}^{\infty} A_{n} \Phi_{n}(r, \theta) e^{-i k \lambda_{n} z}+\sum_{n=0}^{\infty} B_{n} \Phi_{n}(r, \theta) e^{i k \lambda_{n} z}
$$

and

$$
p_{4}(r, \theta, \phi)=\sum_{n=0}^{\infty} C_{n} \Psi_{n}(\theta, \phi) h_{\sigma_{n}}^{(2)}(k r)
$$

respectively. Here, $A_{n} B_{n}$, and $C_{n}$ are modal amplitudes and $k=\omega / c$. The (dimensionless) wavenumber in region $R_{2}$ is given by $\lambda_{n}$, and the eigenfunctions in regions $R_{2}$ and $R_{4}$ are given by $\Phi_{n}(r, \theta)$ and $\Psi_{n}(\theta, \phi)$ respectively. In region $R_{4}, h_{\sigma_{n}}^{(2)}$ is a spherical Hankel function of the second kind, of order $\sigma_{n}$, and a spherical co-ordinate system $(r, \theta, \phi)$ is adopted. The hybrid method proceeds by enforcing continuity of acoustic pressure and normal velocity over surfaces $\Gamma_{A}, \Gamma_{B}$ and $\Gamma_{C}$, and these conditions are enforced here using mode matching ${ }^{21}$. The final system equations are written in matrix form to give

$$
\left[\begin{array}{ll}
\mathbf{R}_{12} & \mathbf{R}_{13} \\
\mathbf{R}_{31} & \mathbf{R}_{34}
\end{array}\right]\left[\begin{array}{l}
\mathbf{T}_{12} \\
\mathbf{T}_{34}
\end{array}\right]=\left[\begin{array}{c}
\mathbf{F}_{1} \\
\mathbf{0}
\end{array}\right]
$$

The individual matrices that make up Eq. (17) are reported in Appendix A. Equation (17) forms a set of $n_{t}\left(n_{t}=n_{1}+2 m_{2}+n_{3}+m_{4}\right)$ linear equations, where $n_{1}$ and $n_{3}$ are the 
number of nodes in regions $R_{1}$ and $R_{3}$, and $m_{2}$ and $m_{4}$ are the number of modes in regions $R_{2}$ and $R_{4}$, respectively.

It is useful first to investigate the sound intensity field in the duct using theoretical predictions before proceeding to make a comparison with measurements; this will illustrate the likely complexity of the sound intensity field and support the discussions that follow. Accordingly, the duct studied here has a radius of $75 \mathrm{~mm}$, with $L_{1}=75 \mathrm{~mm}, L_{2}=5850 \mathrm{~mm}$, and $L_{3}=75 \mathrm{~mm}$, where $L_{1}$ and $L_{2}$ denote the axial length of regions $R_{1}$ and $R_{2}$, respectively, and $L_{3}$ denotes the location of the interface between the modal representation and the finite element discretisation in the duct, see Fig. 2. The sound source is located at $r=65 \mathrm{~mm}$, $\theta=-90^{\circ}$, and $z=-6 \mathrm{~m}$. All three regions of the duct are assumed to contain air, with a speed of sound $c=343.2 \mathrm{~m} / \mathrm{s}$, and density $\rho=1.225 \mathrm{~kg} / \mathrm{m}^{3}$. It is interesting first to visualise the complex sound intensity field inside the duct, especially in the vicinity of the open end to inform the programme of experiments. It is convenient here to use non instantaneous intensity and in Fig. 3 the active and reactive intensities are plotted for a frequency of $1 \mathrm{kHz}$ in the vicinity of the open end of the duct. Here, a streamline vector plot is used in which the length of the vector is proportional to the magnitude of the acoustic intensity component. At $1 \mathrm{kHz}$ only the fundamental mode propagates in the duct and so the problem is axisymmetric. The sound intensity field shown in Fig. 3 is seen to be predominantly active, as the amplitude of the active intensity is clearly larger than that of the reactive intensity. Moreover, it is only very close to the open end of the duct that the active intensity vectors take on a small radial component due to the presence of evanescent modes. This effect is caused by oscillatory behaviour in the sound intensity field and this is seen to be more pronounced for the reactive intensity, which illustrates why the use of complex 
intensity is potentially attractive. The reactive intensity is also seen to be approximately equal to zero at $z \cong-0.045 \mathrm{~m}$; this is the anti-node position of the acoustic pressure so that the end correction for the duct at $1 \mathrm{kHz}$ may roughly be calculated as $(0.0858$ $0.045) / 0.075=0.544$, where $0.0858 \mathrm{~m}$ is a quarter of the wavelength and $0.075 \mathrm{~m}$ is the radius of the duct. This value for the end correction compares to a plane wave calculation of 0.525 and serves to illustrate the influence of the evanescent modes.

Fig. 4 shows the effects of a single higher order propagating mode on the non instantaneous active and reactive intensity fields. Here, a frequency of $1.8 \mathrm{kHz}$ is chosen so that the fundamental and the first circumferential modes are excited in the duct. Fig. 4 shows the complex intensity field for the $r-z$ plane that is coincident with the sound source (so that $\left.\theta=-90^{\circ}\right)$. When a higher order mode is present the intensity field contains strong radial and circumferential components and a circulatory pattern is observed, which repeats itself over the duct length. This behaviour is similar to that observed by Fahy ${ }^{1}$ for active intensity in a two dimensional infinite duct, although Fahy presents mean intensity plots close to a monopole source in order to show the effect of evanescent modes from a source, rather than the change of intensity behaviour caused by the presence of high-order propagating modes near the duct end. The circulatory pattern seen when a higher order mode is present is observed to surround points of minimum acoustic pressure, whereas the regions of maximum acoustic pressure are indicated by regions of divergence in the reactive intensity pattern in the vicinity of the duct wall. But what is important here is the pronounced effect that the higher order mode has on the reactive intensity, which is seen to become much more complicated than the active intensity. Accordingly, if one is attempting to measure the reactive intensity field it is necessary to be able to capture accurately the three dimensional nature of the scattered intensity field. This clearly places significant additional demands on any intensity instrumentation used to undertake these measurements when compared to more conventional 
approaches. Clearly, the theoretical predictions seen in Figs. 3 and 4 illustrate the potential complexity of the intensity sound field, even when only one higher order mode is propagating or when sound is scattered by a simple pipe opening.

\section{Experimental Methodology}

Figure 5 presents a photograph of the experimental setup which was used for the validation of the complex intensity predictions. This setup consists of a flanged PVC duct with a Fané compression driver placed at the closed end of the duct, which is joined to the PVC duct by a short tube of inner diameter $15.4 \mathrm{~mm}$ and length $13 \mathrm{~mm}$. Here, the use of a short tube with a relatively small diameter to connect the loudspeaker to the main duct permits the closed end of the tube to take on the characteristics of an acoustically hard wall, as far as is possible. A tri-axial Microflown USP intensity probe ${ }^{17}$ is used to measure the intensity field close to the open end of the duct. The Microflown 'p-u' probe permits the measurement of all three velocity components simultaneously, the signals obtained by the pressure and velocity sensors may be Fourier transformed and inserted into Eqs. (7) and (8) to give the complex intensity in three orthogonal directions. The probe is supported here by a rigid plastic frame, which enables the position of the probe to be fixed in the axial direction, see Fig. 6. Two frames were constructed, one with the probe positioned in the centre of the duct cross-section and one with the probe located $9 \mathrm{~mm}$ away from the wall of the duct, which permits measurements to be taken at different circumferential locations (at a fixed radius). The probe was orientated in such a way that the three velocity sensors were set to measure the axial, radial and circumferential velocity components. The frame was designed to minimise disturbance of the sound intensity field, whilst still providing sufficient stability when supporting the probe. A National Instruments DAQ NI PXIE-6358 system was used to 
acquire the signals from the USP probe. This system was controlled with LabVIEW software and was designed to generate acoustic stimulus and synchronously record the USP probe signals at a sampling rate of $48 \mathrm{kHz}$.

The Microflown USP intensity probe was chosen here because it is the smallest device available on the market for measuring the three components of the acoustic velocity vector over a broad audio frequency range. The device was calibrated using calibration formulae provided by Microflown. Generally, this calibration procedure yields accurate results. However, at very low or high frequencies when the sound field is either strongly active or reactive, the measurement accuracy strongly depends on the accuracy of the phase calibration $^{22}$. Furthermore, the majority of the Microflown calibration reports published so far in the literature are based on measuring intensity in one direction only ${ }^{19,22,23}$. The only calibration investigation available for a ' $p$ - $u$ ' probe measuring pressure and velocities in three orthogonal directions simultaneously is provided by the manufacturer ${ }^{17}$. Accordingly, in the experiments carried out here, the frequency range of the sound source is limited to between $800 \mathrm{~Hz}$ and $2000 \mathrm{~Hz}$ in the expectation that acceptable accuracy can be obtained without further calibration tests.

\section{COMPARSION BETWEEN PREDICTION AND EXPERIMENT}

Comparisons between prediction and measurement are carried out here both for instantaneous and non-instantaneous complex intensity, although for instantaneous intensity a steady state sound field is examined. In Fig. 7 the instantaneous active and reactive intensities are presented at the exit from the duct $(r=0, z=0)$ for a steady state sound field. Here, the measured and predicted axial intensity is compared using the methods of Heyser ${ }^{7}$ and Stanzial and $\operatorname{Prodi}^{10}$ for sinusoidal excitation at a frequency of $1 \mathrm{kHz}$, which is chosen in 
order to restrict sound propagation to plane waves. The difference in the two representations of instantaneous intensity is clearly evident in Fig. 7, and here the method of Heyser ${ }^{7}$ delivers a running average for the active intensity and an upper envelope for the reactive intensity.

This is similar to the behaviour observed in section II. In contrast, the method of Stanzial and Prodi $^{10}$ is capable of closely following the theoretical predictions once the signal has achieved a steady state, with a maximum relative error in the active intensity of $5 \%$ in the region above $2 \mathrm{~ms}$. Note that in the region of approximately $0-2 \mathrm{~ms}$ the system response is transient and so one cannot expect to see agreement between prediction and measurement in this region because the predictions are based on a time stationary sound field. Accordingly, one may observe here that the method of Stanzial and Prodi is accurate under plane wave propagation conditions when the sound field is time stationary, and this supports the original findings of Schiffrer and Stanzial ${ }^{8}$.

The non-instantaneous axial active and reactive intensity is shown as a function of the axial coordinate $z$ in Fig. 8 for an excitation frequency of $1 \mathrm{kHz}$ (plane wave). It is evident in Fig. 8(a) that away from the duct exit plane the theoretical active intensity is almost constant, although near to the duct end active intensity amplitude reduces whereas the reactive intensity increases. This behaviour can also be observed in Fig. 3. In Fig. 8 good agreement is generally observed between prediction and measurement, with an error of less than about $10 \%$, although the measured active intensity exhibits some oscillatory behaviour and deviates from the theoretical predictions as the distance to the open end of the duct increases. In general, the results presented in Fig. 7 illustrate that it is possible to obtain agreement to within 5\% for the predicted and measured data for the non instantaneous complex intensity under plane wave propagation conditions. 
The results presented in Fig. 4 do, however, suggest that the complex intensity distribution should be far more complex when at least one higher-order mode is excited in the duct. Figures 9-11 show the predicted and measured data for the non instantaneous complex intensity with a monochromatic sound field at a frequency of $1.8 \mathrm{kHz}$. At this frequency, one circumferential mode propagates in addition to the fundamental (plane wave) mode. In this experiment, the centre of the tube connecting the output of the Fané compression driver was installed $9 \mathrm{~mm}$ from the duct wall at $\theta_{0}=-90^{\circ}$ (see Fig. 1), and the Microflown probe was placed inside the duct at $z=-0.2 \mathrm{~m}$ and $9 \mathrm{~mm}$ away from the duct wall. Data for the axial, circumferential and radial intensity vector components were then obtained for different circumferential locations by rotating the probe frame through a full circle and taking measurements every $10^{\circ}$.

Figs. 9-11 illustrate that when a multi-modal sound field is present in the duct, it becomes increasingly difficult to obtain good agreement between prediction and measurement. The dominant components in this sound field are the axial and circumferential intensity and here the agreement between the behaviour in the amplitude of the predicted and measured axial active intensities in Fig. 9(a) is generally good but the pattern in the measured data is shifted by approximately $20^{\circ}$ with respect to the predicted result. The relative error between the amplitudes of the maxima in the predicted and measured active intensity (axial component) is relatively small, being less than $1 \%$, but this error increases significantly when the value of the circumferential coordinate $\theta$ is less than $120^{\circ}$, and here the amplitude of the active axial intensity becomes relatively small. This suggests that the probe is able to capture well the qualitative behaviour of the sound energy radiating from the pipe, but not its exact amplitude at a given circumferential coordinate. There is large a discrepancy, moreover, between the theoretical and experimental axial reactive intensities for circumferential coordinates greater 
than $50^{\circ}$, which may be caused by the fact that most of the energy is radiated from the duct at $1.8 \mathrm{kHz}$, making accurate measurements with the $p$-u probe more challenging. Figure 10 shows that the reactive part of the circumferential intensity component oscillates over the pipe cross-section and predicted behaviour generally agrees well with the measurement. However, one again observes a shift between the measured and predicted intensities, this time of about $15^{\circ}$. The measurement of the propagating (active) part of the circumferential intensity component is much less accurate and it does not match the prediction even in terms of its sign. Here, one expects a symmetric intensity field distribution and so this should not be entirely negative. It is likely that some scattering from the frame supporting the probe has affected these measurements and that this effect is more pronounced in the case of the circumferential velocity component, especially at the higher frequencies and/or when higherorder modes are excited. Finally, Fig. 11 shows the radial complex intensity. This intensity vector component is generally smaller than the axial and circumferential components seen in Figs. 9 and 10. Good agreement between the predicted and measured active and reactive intensities is observed in the case of the radial intensity component (see Fig. 11) and this represents an improvement when compared to the errors in the measured data for the axial and circumferential intensities as shown in Figs. 9 and 10. This improvement may be explained by a reduction in the influence of the supporting frame and the probe body when taking measurements in the radial direction and more accurate response of the radially orientated velocity sensor in the USP probe.

The comparison between prediction and measurement in Figs. 9-11 demonstrates that obtaining good agreement between measured and predicted complex intensity presents a significant challenge when using a tri-axial intensity probe, even in the case of a well-defined problem such as an open ended duct. Clearly, it is possible to obtain good agreement 
between prediction and measurement for at least four out of the six complex intensity characteristics presented in Fig. 9-11. However, the agreement between the measured and predicted axial reactive and circumferential active intensities is poor. It is possible that these problems are caused by an increase in sound scattering from the supporting frame and the body of the probe as the frequency of sound is increased. Alternatively, it is also possible that the tri-axial probe finds it more difficult to resolve accurately all three complex intensity components at higher frequencies and/or under multi-modal conditions because of errors in the acoustic velocity measurements. Thus, it is clear that accurately measuring all the three intensity components in a complex sound field within a duct is a challenge, and in view of this caution should be exercised when interpreting complex intensity measurements obtained when higher order modes are propagating in a duct.

These measurements illustrate the general difficulty of measuring accurately the complex intensity vector and here difficulties have been observed for a time stationary sound field in which only limited scattering from the open end of the duct is present. It is expected, therefore, that further difficulties would be encountered for transient scattering problems. Thus, even for a relatively simple and well defined experimental set up one can expect only a limited quantitative understanding of a scattered sound field based on complex intensity measurements. This is because the reactive sound field is typically very complicated under those conditions of interest and current measurement techniques are not sufficiently accurate to allow one to satisfactorily resolve the complex intensity vector. This has important ramifications for the use of complex intensity in sound scattering problems, especially if one is attempting to apply inverse analysis techniques in an attempt to recover information about the sound source. 


\section{CONCLUSIONS}

Complex intensity has been studied here for a relatively well defined problem that encompasses sound scattering from the open end of a duct, as well as the propagation of plane and higher order modes inside the duct. For instantaneous intensity a comparison between the methods of Heyser ${ }^{7}$ and Stanzial and Prodi ${ }^{10}$ demonstrates that Heyser's method always delivers a consistent representation of the energy transport within a duct. However, the method of Heyser is seen only to deliver a running time average for the instantaneous active intensity, as well as the upper envelope of the instantaneous reactive intensity. The method of Stanzial and Prodi ${ }^{10}$ adopts a very different approach and for steady state sound fields it is shown that the method delivers a much more detailed representation of complex intensity when compared to the method of Heyser. Moreover, for sound radiated by an open ended duct in a time stationary problem the method of Stanzial and Prodi agrees very well with theoretical predictions under plane wave condtions. However, for transient problems some difficulties are observed with the method of Stanzial and Prodi ${ }^{10}$ and these are linked to the time averaging procedure that appears in their definitions of radiating and oscillating intensity. Following the use of simple theoretical calculations it is demonstrated that if different sound signals, or in this case the predicted incident and reflected pulse in a duct, are included within the time averaging procedure then some cancellation between the signals is possible. This delivers erroneous predictions for plane wave propagation, which may be rectified only by windowing out individual signals. Accordingly, it is concluded here that caution should be exercised when applying Stanzial and Prodi's approach to transient problems, especially when studying the acoustic near field of complex sound sources in which multiple equivalent noise sources are present. 
It is shown here that it is possible to obtain good quantitative agreement between predicted and measured complex intensity provided one studies a very simple problem, which in this case necessitated the use of plane waves in the duct. Here, good agreement in a time stationary sound field is observed both at the exit of an open ended duct, and within the duct itself. However, when the sound field is complicated by adding a higher order mode, it is found to be much more difficult to obtain good agreement between prediction and experiment. It is seen that the reactive sound intensity field becomes significantly more complicated than the active sound intensity field (the one traditionally measured) and this places a much more stringent requirement on the accuracy of the measuring equipment. Under carefully controlled conditions it was found to be possible to obtain good agreement between prediction and measurement for four out of six of the tri-axial components of non instantaneous complex intensity, but the active circumferential and reactive axial intensities exhibit significant discrepancies. Problems are thought to be caused by the interaction between the sound field and the supporting frame, as well as difficulties with accurately calibrating the Microflown device. However, it is also thought that current measurement techniques are simply unable to resolve the complex nature of a reactive sound field at accuracy sufficient for delivering reliable quantitative data, and this was observed even for a relatively simple problem.

Complex intensity has the potential to deliver additional information about the nature of energy propagation in the near field of a radiating sound source, or alternatively in a multi modal environment within a duct. The accurate measurement of the instantaneous and noninstantaneous complex intensity is, however, difficult to achieve with existing instrumentation. Technology that is currently available for measuring intensity can only be 
relied upon to provide a qualitative investigation into a complex intensity sound field. This is likely to limit the effectiveness of any investigation into a radiated sound field using an inverse analysis approach that is based on the use of reactive intensity.

\section{ACKNOWLEDGEMENTS}

The authors would like to thank the UK Engineering and Physical Sciences Research Council (Grant numbers EP/H015280/1 and EP/H015469) for their support of the work reported in this article. The authors are grateful to the technical staff at the University of Bradford, and in particular, Mr. Nigel Smith, for support in building the experimental rig. 


\section{APPENDIX A}

$$
\begin{aligned}
& \mathbf{R}_{\mathbf{1 2}} \mathbf{T}_{\mathbf{1 2}}=\left[\begin{array}{cccc}
\mathbf{G}_{\mathbf{1 A A}} & \mathbf{G}_{\mathbf{1 A e}} & \mathbf{Q}_{1}^{T} & -\mathbf{Q}_{1}^{T} \mathbf{D}_{\mathbf{1}} \\
\mathbf{G}_{\mathbf{1 e A}} & \mathbf{G}_{1 \mathrm{ee}} & \mathbf{0} & \mathbf{0} \\
\mathbf{Q}_{\mathbf{1}} & \mathbf{0} & -\mathbf{M}_{\mathbf{1}} & -\mathbf{M}_{\mathbf{1}} \mathbf{D}_{\mathbf{1}} \\
\mathbf{0} & \mathbf{0} & -\mathbf{M}_{\mathbf{3}} \mathbf{D}_{\mathbf{1}} & -\mathbf{M}_{\mathbf{3}}
\end{array}\right]\left[\begin{array}{c}
\mathbf{p}_{\mathbf{1 A}} \\
\mathbf{p}_{\mathbf{1 e}} \\
\boldsymbol{A} \\
\widetilde{B}
\end{array}\right], \\
& \mathbf{R}_{13} \mathbf{T}_{34}=\left[\begin{array}{cccc}
\mathbf{0} & \mathbf{0} & \mathbf{0} & \mathbf{0} \\
\mathbf{0} & \mathbf{0} & \mathbf{0} & \mathbf{0} \\
\mathbf{0} & \mathbf{0} & \mathbf{0} & \mathbf{0} \\
\mathbf{Q}_{3} & \mathbf{0} & \mathbf{0} & \mathbf{0}
\end{array}\right]\left[\begin{array}{c}
\mathbf{p}_{3 \mathrm{~B}} \\
\mathbf{p}_{3 \mathrm{e}} \\
\mathbf{p}_{3 \mathrm{C}} \\
\widetilde{C}
\end{array}\right] \\
& \mathbf{R}_{31} \mathbf{T}_{12}=\left[\begin{array}{cccc}
0 & 0 & -Q_{3}^{T} \mathbf{D}_{1} & Q_{3}^{T} \\
0 & 0 & 0 & 0 \\
0 & 0 & 0 & 0 \\
0 & 0 & 0 & 0
\end{array}\right]\left[\begin{array}{c}
\mathbf{p}_{1 \mathrm{~A}} \\
\mathbf{p}_{1 \mathrm{e}} \\
\boldsymbol{A} \\
\widetilde{B}
\end{array}\right] \\
& \mathbf{R}_{34} \mathbf{T}_{\mathbf{3 4}}=\left[\begin{array}{cccc}
\mathbf{G}_{3 \mathrm{BB}} & \mathbf{G}_{3 \mathrm{BBe}} & \mathbf{G}_{3 \mathrm{BC}} & \mathbf{0} \\
\mathbf{G}_{3 \mathrm{eB}} & \mathbf{G}_{3 \mathrm{ee}} & \mathbf{G}_{3 \mathrm{eC}} & \mathbf{0} \\
\mathbf{G}_{3 \mathrm{BB}} & \mathbf{G}_{3 \mathrm{Ce}} & \mathbf{G}_{3 \mathrm{CC}} & -\mathbf{Q}_{4}^{T} \\
\mathbf{0} & \mathbf{0} & \mathbf{Q}_{\mathbf{4}} & -\mathbf{M}_{\mathbf{4}} \mathbf{D}_{\mathbf{4}}^{-1}
\end{array}\right]\left[\begin{array}{c}
\mathbf{p}_{3 \mathrm{~B}} \\
\mathbf{p}_{3 \mathrm{e}} \\
\mathbf{p}_{3 \mathrm{C}} \\
\widetilde{\boldsymbol{C}}
\end{array}\right] \\
& \mathbf{F}_{\mathbf{1}}=\left[\mathbf{N}_{\mathbf{1}}^{\mathbf{T}}\left(r_{0}, \theta_{0}, z_{0}\right) \quad \mathbf{0} \quad \mathbf{0} \quad \mathbf{0}\right]^{\mathbf{T}} .
\end{aligned}
$$

Here, $\left[\boldsymbol{D}_{\mathbf{1}}\right]$ and $\left[\boldsymbol{D}_{\mathbf{4}}\right]$ are diagonal matrices with each diagonal element given by $e^{-i k \lambda_{n} L}$, $\left(n=0,1, \cdots, m_{2}\right)$, and $\partial h_{\sigma_{n}}^{(2)}(k r) /\left.\partial \mathrm{r}\right|_{r=R},\left(n=0,1, \cdots, m_{4}\right)$, respectively. The modal amplitude coefficients are normalised as $\mathbf{D}_{\mathbf{1}}^{-\mathbf{1}} \mathbf{B}=\widetilde{\mathbf{B}}$ and $\mathbf{D}_{4} \mathbf{C}=\tilde{\mathbf{C}}$. The constituent matrices are given by

$$
\left[\mathbf{G}_{\mathbf{1}}\right]=\int_{\Omega_{1}}\left[\nabla \mathbf{N}_{\mathbf{1}}^{\mathrm{T}} \nabla \mathbf{N}_{\mathbf{1}}-k^{2} \mathbf{N}_{\mathbf{1}}^{\mathbf{T}} \mathbf{N}_{\mathbf{1}}\right] d \Omega_{1}
$$




$$
\begin{gathered}
{\left[\mathbf{G}_{3}\right]=\int_{\Omega_{3}}\left[\nabla \mathbf{N}_{\mathbf{3}}^{\mathbf{T}} \nabla \mathbf{N}_{\mathbf{3}}-k^{2} \mathbf{N}_{\mathbf{3}}^{\mathbf{T}} \mathbf{N}_{\mathbf{3}}\right] d \Omega_{3}} \\
{\left[\mathbf{Q}_{\mathbf{1}}\right]=i k \lambda_{m} \int_{\Gamma_{\mathrm{A}}} \Phi_{m} \mathbf{N}_{\mathbf{1}} d \Gamma_{\mathrm{A}}, \quad\left(m=0,1, \cdots, m_{2}\right)} \\
{\left[\mathbf{Q}_{\mathbf{3}}\right]=i k \lambda_{m} \int_{\Gamma_{\mathrm{B}}} \Phi_{m} \mathbf{N}_{\mathbf{3}} d \Gamma_{\mathrm{B}}, \quad\left(m=0,1, \cdots, m_{2}\right)} \\
{\left[\mathbf{Q}_{\mathbf{4}}\right]=\int_{\Gamma_{\mathrm{C}}} \Psi_{m} \mathbf{N}_{\mathbf{3}} d \Gamma_{\mathrm{C}}, \quad\left(m=0,1, \cdots, m_{4}\right)} \\
{\left[\mathbf{M}_{\mathbf{1}}\right]=i k \lambda_{m} \int_{\Gamma_{\mathrm{A}}} \Phi_{m} \Phi_{n} d \Gamma_{\mathrm{A}}, \quad\left(m=0,1, \cdots, m_{2} ; n=0,1, \cdots, m_{2}\right)} \\
{\left[\mathbf{M}_{3}\right]=i k \lambda_{m} \int_{\Gamma_{\mathrm{B}}} \Phi_{m} \Phi_{n} d \Gamma_{\mathrm{B}}, \quad\left(m=0,1, \cdots, m_{2} ; n=0,1, \cdots, m_{2}\right)} \\
{\left[\mathbf{M}_{\mathbf{4}}\right]=h_{\sigma_{n}}^{(2)}(k R) \int_{\Gamma_{\mathrm{C}}} \Psi_{m} \Psi_{\mathrm{n}} d \Gamma_{\mathrm{C}}, \quad\left(m=0,1, \cdots, m_{4} ; n=0,1, \cdots, m_{4}\right)}
\end{gathered}
$$

Here, the outer radius of the finite element mesh in region $R_{3}$ is $R=150 \mathrm{~mm}$. If only plane waves propagate, then the numerical model reduces from three to two dimensions, but when higher order modes propagate (above $1341 \mathrm{~Hz}$ for this duct) the problem is no longer axisymmetric and a three dimensional model is necessary. For the two dimensional model, eight noded quadrilateral isoparametric elements were used to discretise region $R_{1}$, and the part of region $R_{3}$ which lies inside the duct. In the case of the part of region $R_{3}$ that lies outside of the duct, six noded triangular isoparametric elements were used. For the three dimensional model, ten noded tetrahedral isoparametric elements are used to discretise regions $R_{1}$ and $R_{3}$, and six noded triangular isoparametric elements are used for the surface of regions $R_{1}$ and $R_{3}$. A very fine mesh is used in regions $R_{1}$ and $R_{3}$ in order to ensure accuracy of the calculations. A minimum of 45 nodes per wavelength in the two dimensional model is chosen. For the three dimensional model, the element size within $R_{3}$ is optimised so that it is finer on the surfaces $\Gamma_{\mathrm{B}}$ and $\Gamma_{\mathrm{C}}$ (see Fig. 1) and coarser within the volume of $R_{3}$ in order to 
improve the accuracy of the integration over each surface, but also to control the number of elements. Here, at least 27 nodes per wavelength are used on surfaces $\Gamma_{B}$ and $\Gamma_{C}$, whilst a minimum of 11 nodes per wavelength is used within the volume of regions $R_{1}$ and $R_{3}$. For the two dimensional model, 40 modes are used in region $R_{2}$ and 80 modes in region $R_{4}$. For the three dimensional model, 60 modes are used in region $R_{2}$ and 120 modes in region $R_{4}$. 


\section{REFERENCES}

${ }^{1}$ F. J. Fahy, "Sound intensity," (E \& FN Spon, London, 1995) pp.38-99.

2 J. A. Mann and J. Tichy, "Near-field identification of vibration sources, resonant cavities, and diffraction using acoustic intensity measurements," J. Acoust. Soc. Am. 90(2), 720-729 (1991).

${ }^{3}$ J. A. Mann and J. Tichy, “Acoustic intensity analysis: Distinguishing energy propagation and wave-front propagation ” J. Acoust. Soc. Am. 90(1), 20-25 (1991).

${ }^{4}$ N. Prodi and D. Stanzial, "A novel intensimetric technique for monitoring the radiative properties of sound fields," J. Audio Eng. Soc. 47(5), 363-372 (1999).

${ }^{5}$ R. J. Barton, K. B. Smith, and I. H. T. Vincent, "Characterization of scattered acoustic intensity fields in the resonance region of a motionless rigid sphere," J. Acoust. Soc. Am. 127(6), EL240-EL245 (2010).

${ }^{6}$ F. Jacobsen, "A note on instantaneous and time-averaged active and reactive sound intensity,” J. Sound Vib. 147(3), 489-496 (1991).

${ }^{7}$ R. C. Heyser, "Instantaneous intensity," AES $81^{\text {st }}$ Convention, Audio Engineering Society, California, preprint 2399 (1986).

${ }^{8}$ G. Schiffrer and D. Stanzial, "Energetic properties of acoustic fields,” J. Acoust. Soc. Am. 96(6), 3645-3653 (1994).

${ }^{9}$ D. Stanzial, N. Prodi, and G. Schiffrer, "Reactive acoustic intensity for general fields and energy polarization,” J. Acoust. Soc. Am. 99(4), 1868-1876 (1996).

${ }^{10}$ D. Stanzial and N. Prodi, "Measurements of newly defined intensimetric quantities and their physical interpretation,” J. Acoust. Soc. Am. 102(4), 2033-2039 (1997).

${ }^{11}$ D. Stanzial, G. Sacchi, and G. Schiffrer, "On the physical meaning of the power factor in acoustics,” J. Acoust. Soc. Am. 131(1), 269-280 (2012). 
${ }^{12}$ J. A. Mann, J. Tichy, and A. J. Romano, "Instantaneous and time-averaged energy transfer in acoustic fields," J. Acoust. Soc. Am. 82(1), 17-30 (1987).

${ }^{13}$ B. R. Rapids and G. C. Lauchle, "Vector intensity field scattered by a rigid prolate spheroid,” J. Acoust. Soc. Am. . 120(1), 38-48 (2006).

${ }^{14}$ R. J. Barton, K. B. Smith, and H. T. Vincent, "A characterization of the scattered acoustic intensity field in the resonance region for simple spheres," J. Acoust. Soc. Am. 129(5), 27722784 (2011).

${ }^{15}$ F. Jacobsen and A. R. Molares, "Ensemble statistics of active and reactive sound intensity in reverberation rooms," J. Acoust. Soc. Am. . 129, (1), 211-218 (2011).

${ }^{16}$ C. E. Dean and J. P. Braselton, "Visualization of the energy flow for elastic waves: comparison and contrast of conventional vector field vs. color coded representation of the poynting vector," in Theoretical and computational acoustics 2003, World Scientific, Hawaii. p. 78-90 (2003).

${ }^{17}$ www.microflown.com. Date last viewed 11/6/13.

${ }^{18}$ H.-E. d. Bree, “The microflown: An acoustic particle velocity sensor,” Acoust. Aust. 31, 91-94 (2003).

${ }^{19}$ F. Jacobsen and V. Jaud, "A note on the calibration of pressure-velocity sound intensity probes" J. Acoust. Soc. Am. 120(2), 830-837 (2006).

${ }^{20}$ W. Duan and R. Kirby, “A hybrid finite element approach to modeling sound radiation from circular and rectangular ducts,” J. Acoust. Soc. Am. 131(5), 3638-3649 (2012).

${ }^{21}$ R. Kirby, "Modeling sound propagation in acoustic waveguides using a hybrid numerical method,” J. Acoust. Soc. Am. 124(4), 1930-1940 (2008).

${ }^{22}$ D. Stanzial, G. Sacchi, and G. Schiffrer, "Calibration of pressure-velocity probes using a progressive plane wave reference field and comparison with nominal calibration filters," J. Acoust. Soc. Am. 129(6), 3745-3755 (2011). 
${ }^{23}$ T. G. H. Basten and H.-E. d. Bree, "Full bandwidth calibration procedure for acoustic probes containing a pressure and particle velocity sensor” J. Acoust. Soc. Am. 127(1), 264270 (2010). 


\section{FIGURE CAPTIONS}

Fig. 1. Instantaneous active (a) and reactive (b) intensity at $200 \mathrm{~Hz} . \_$, predictions using on Stanzial and Prodi's method ${ }^{10} ;----$-, predictions based on Heyser's method ${ }^{7}$

Fig. 2. Geometry of open ended duct.

Fig. 3. Active (a) and reactive (b) intensity at $1000 \mathrm{~Hz}$.

Fig. 4. Active (a) and reactive (b) intensity at $1800 \mathrm{~Hz}$.

Fig. 5. A photograph of the experimental setup.

Fig. 6. The USP probe installed in the frame.

Fig. 7. Instantaneous complex intensity at $1000 \mathrm{~Hz}$ : instantaneous active (a) and instantaneous reactive (b): $\longrightarrow$, theory; $-ー-$, experimental data based on Stanzial and Prodi's $\operatorname{method}^{10} ;----$ - experimental data based on Heyser's method ${ }^{7}$.

Fig. 8: Active (a) and reactive (b) axial intensity at 1000Hz: — theory; $\boldsymbol{\Lambda}$, experiment.

Fig. 9: Active (a) and reactive (b) axial intensity at $1800 \mathrm{~Hz}:-$, theory; $\boldsymbol{\Lambda}$, experiment.

Fig. 10: Active (a) and reactive (b) circumferential intensity at $1800 \mathrm{~Hz}:-\longrightarrow$, theory; experiment. 
Fig. 11: Active (a) and reactive (b) radial intensity at $1800 \mathrm{~Hz}:-$, theory; $\mathbf{\Lambda}$, experiment. 

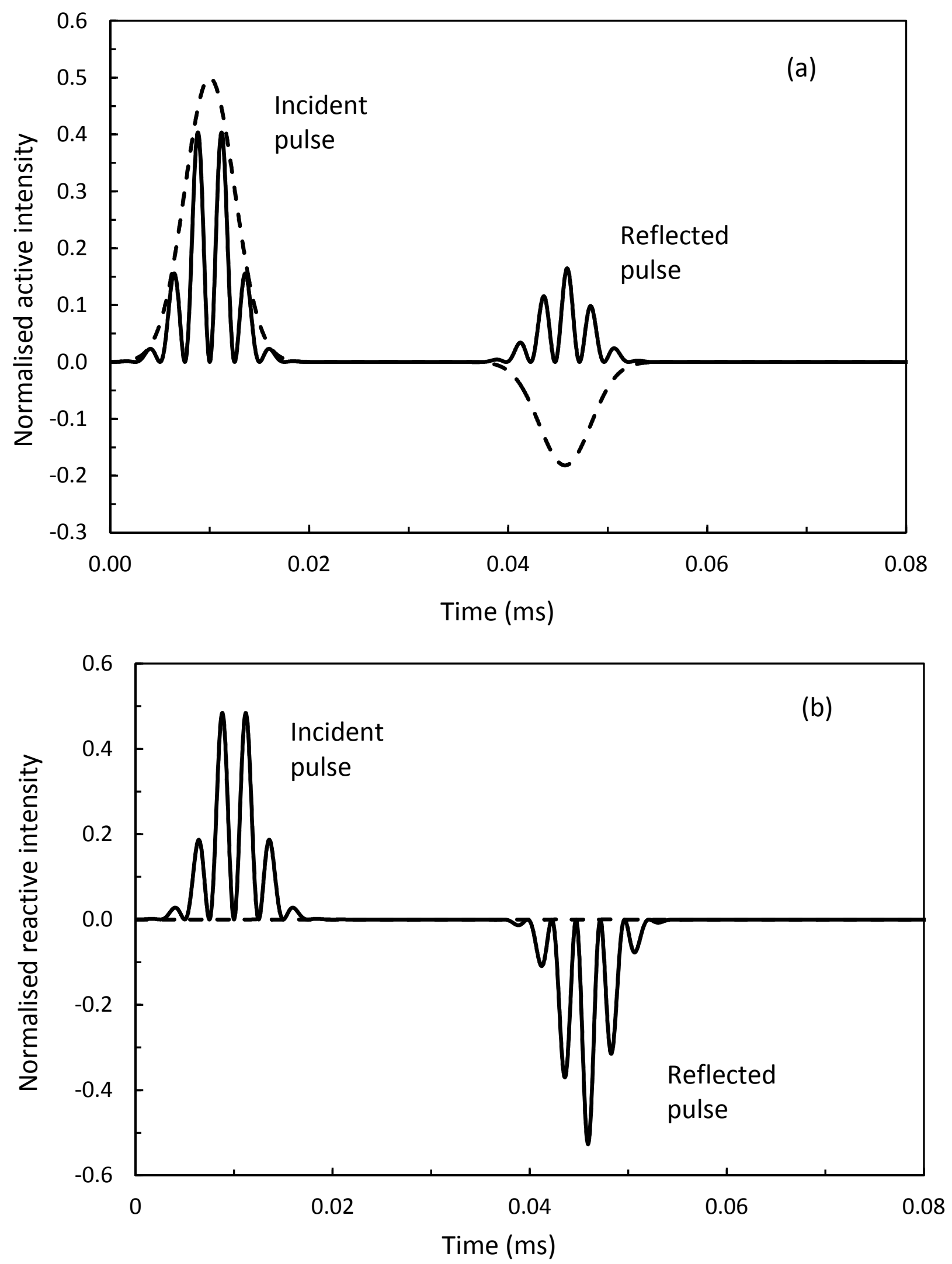

Fig. 1. Instantaneous active (a) and reactive (b) intensity at $200 \mathrm{~Hz}$.

predictions using on Stanzial and Prodi's method ${ }^{10}$; - - - - , predictions based on Heyser's method ${ }^{7}$ 


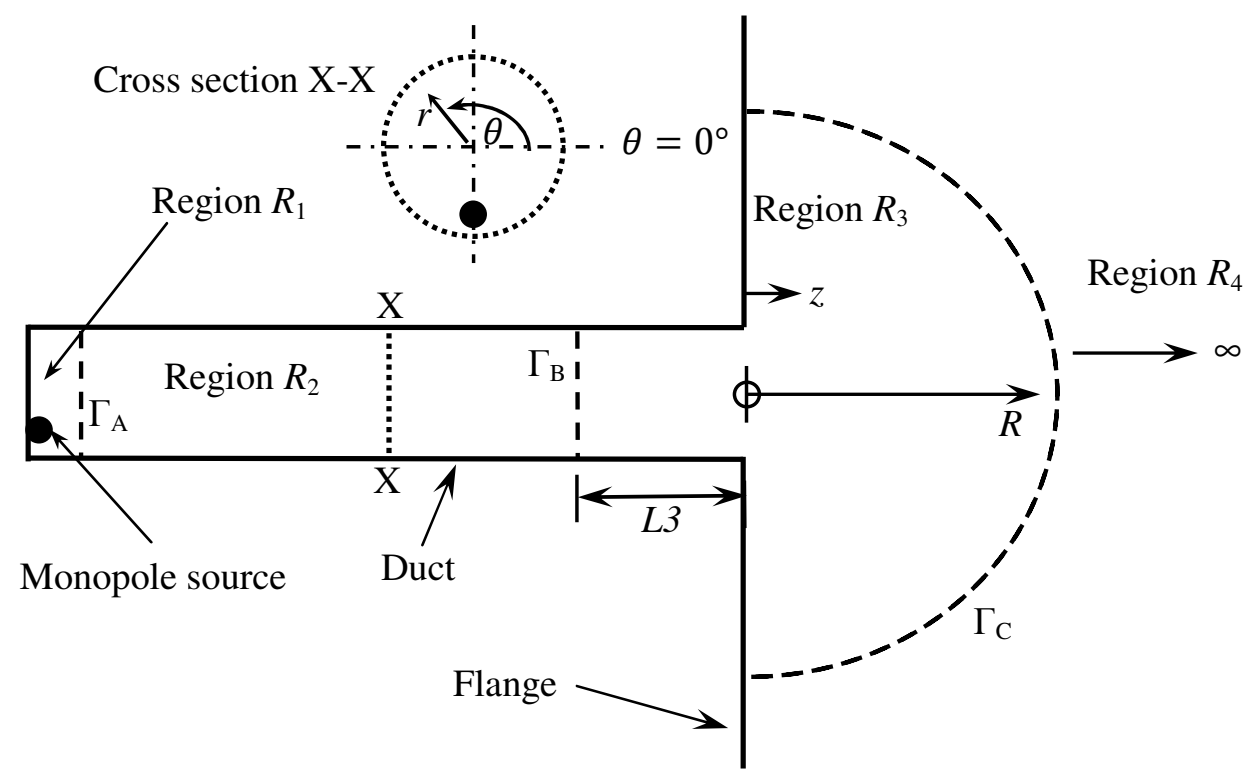

Fig. 2. Geometry of duct. 

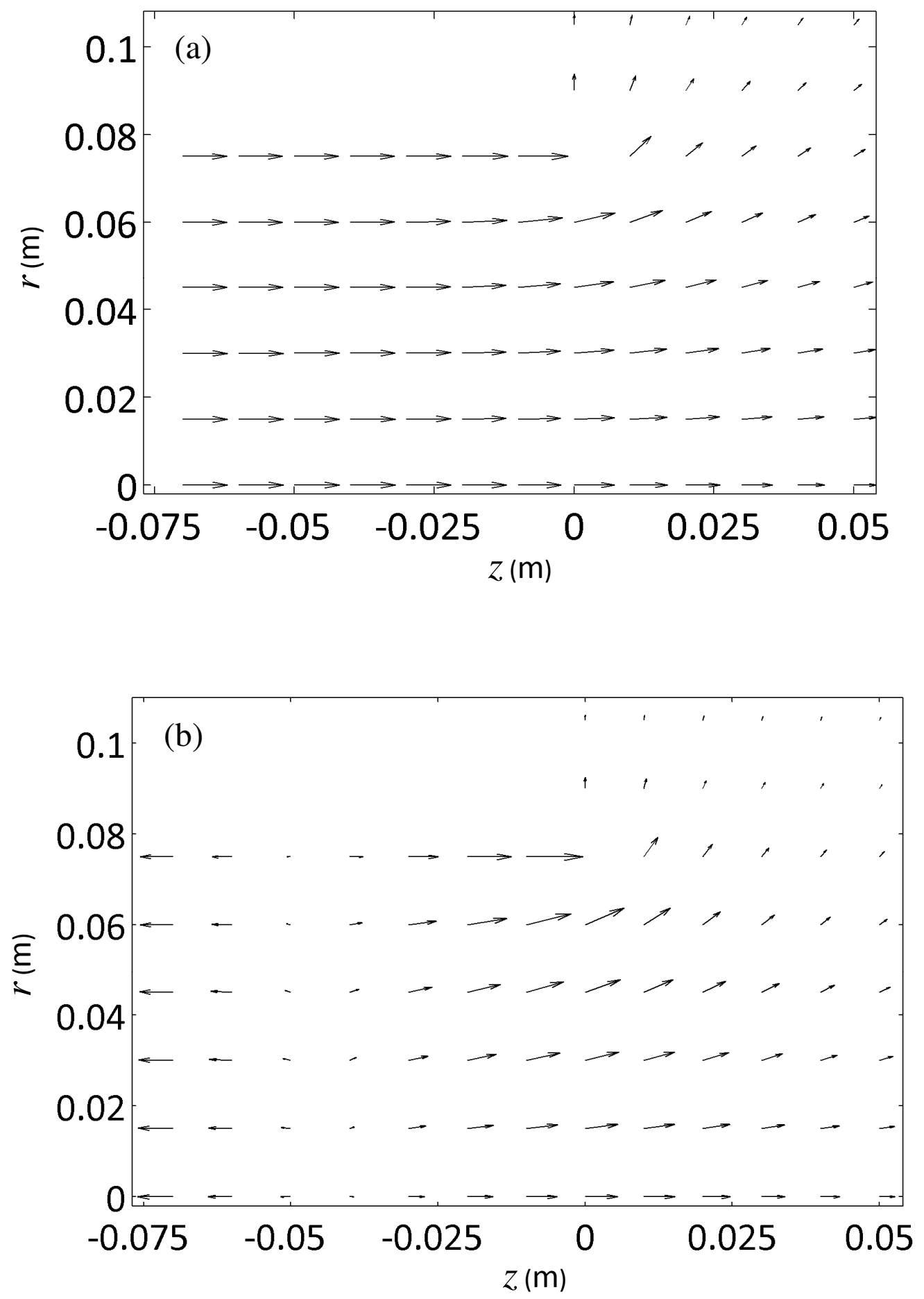

Fig. 3. Active (a) and reactive (b) intensity at $1000 \mathrm{~Hz}$. 

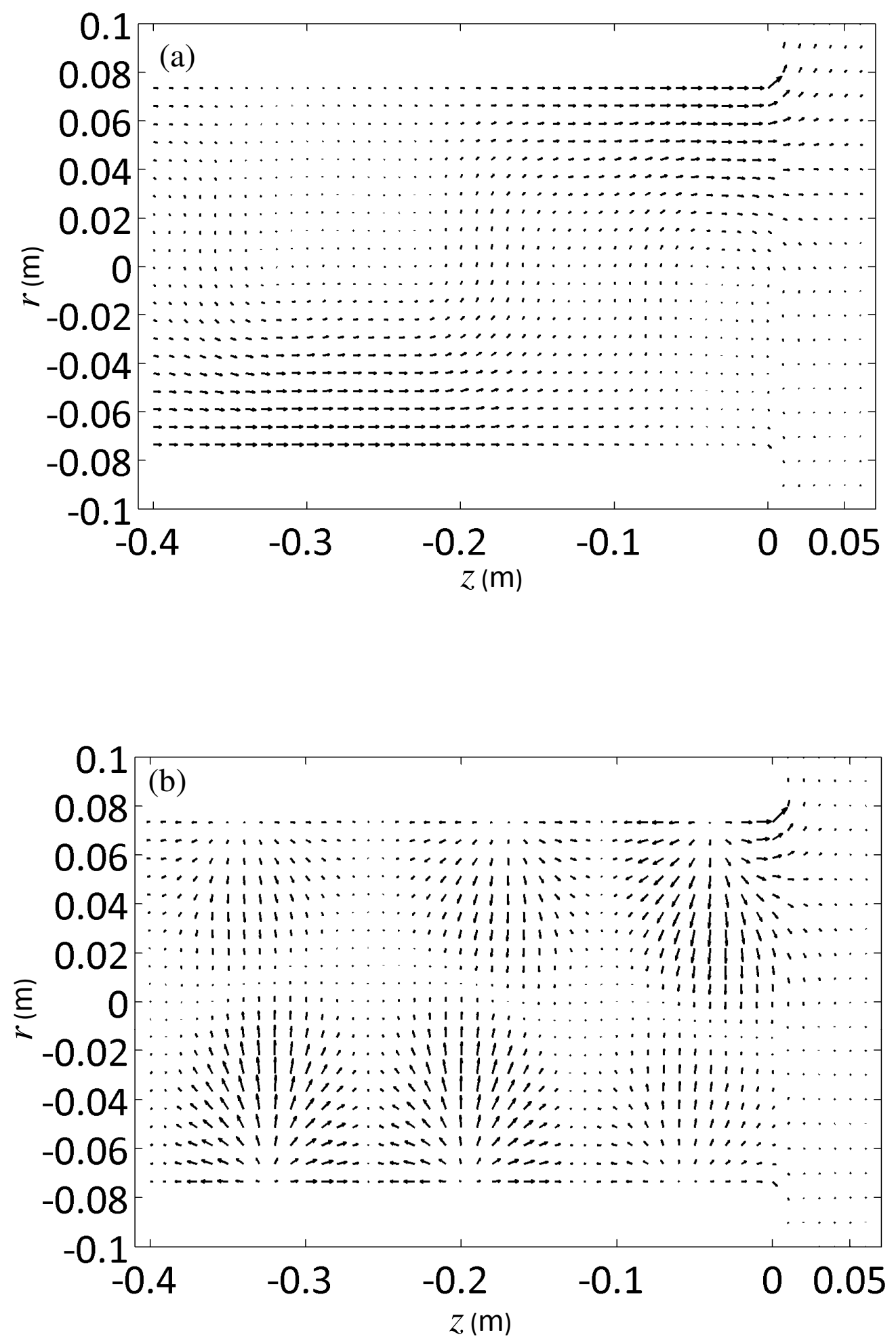

Fig. 4. Active (a) and reactive (b) intensity at $1800 \mathrm{~Hz}$. 


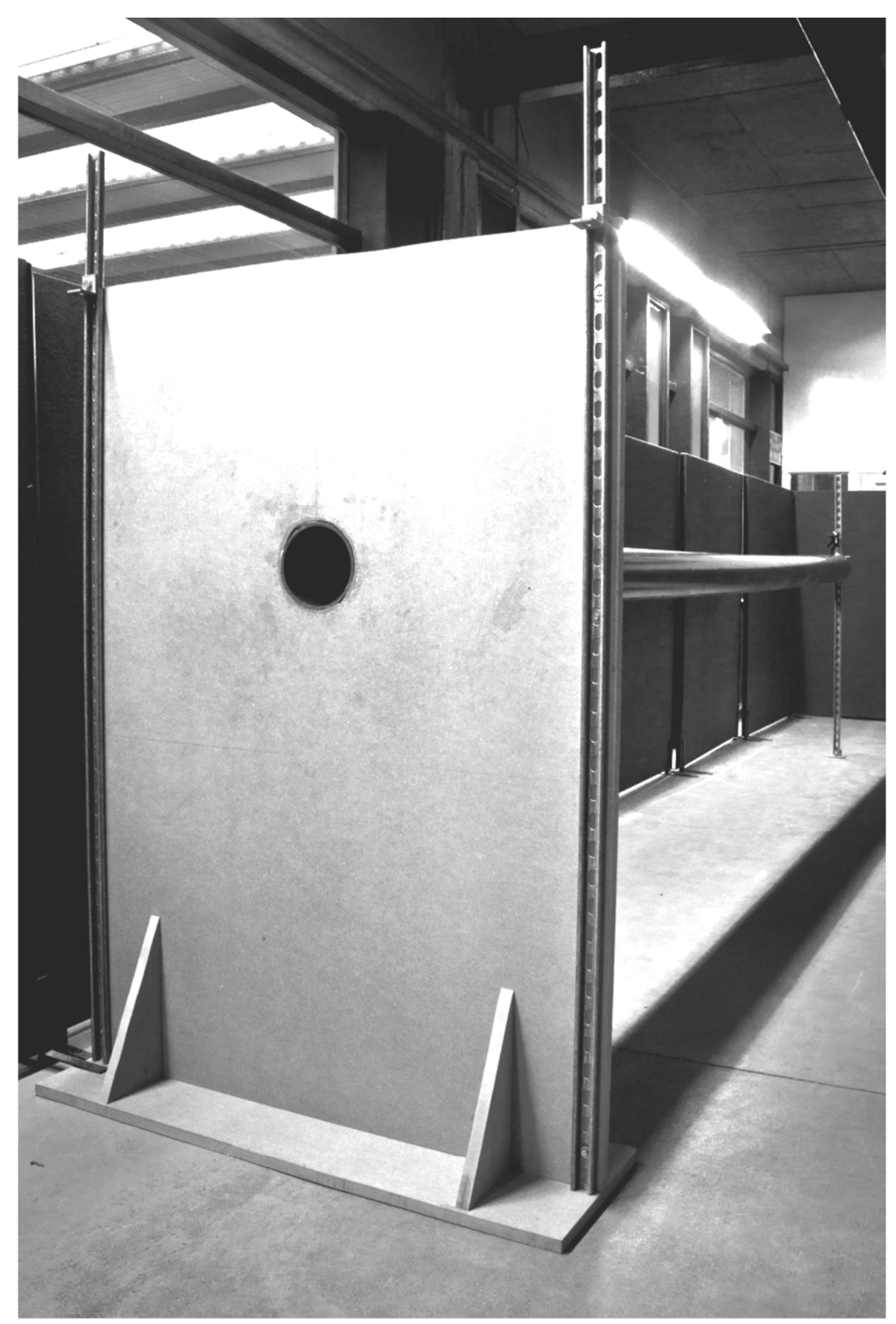

Fig. 5. A photograph of the experimental setup. 


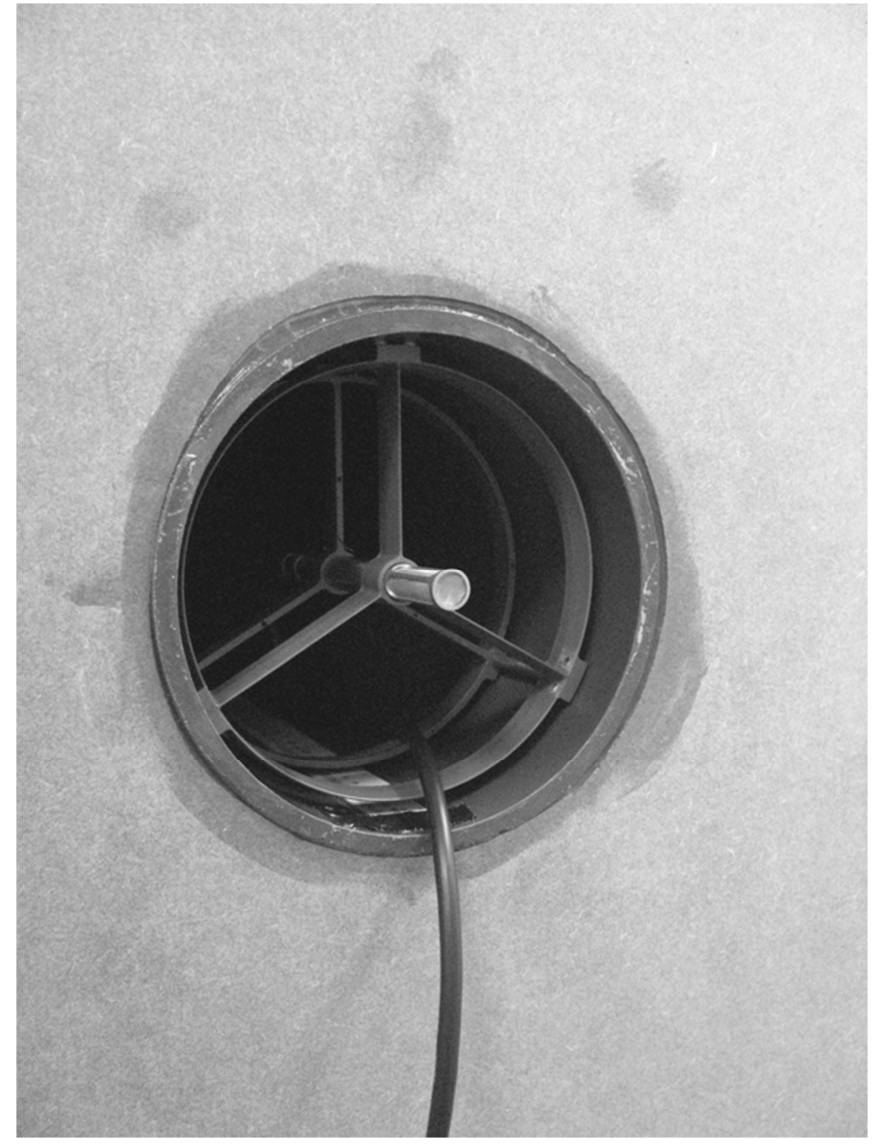

Fig. 6. The USP probe installed in the frame. 

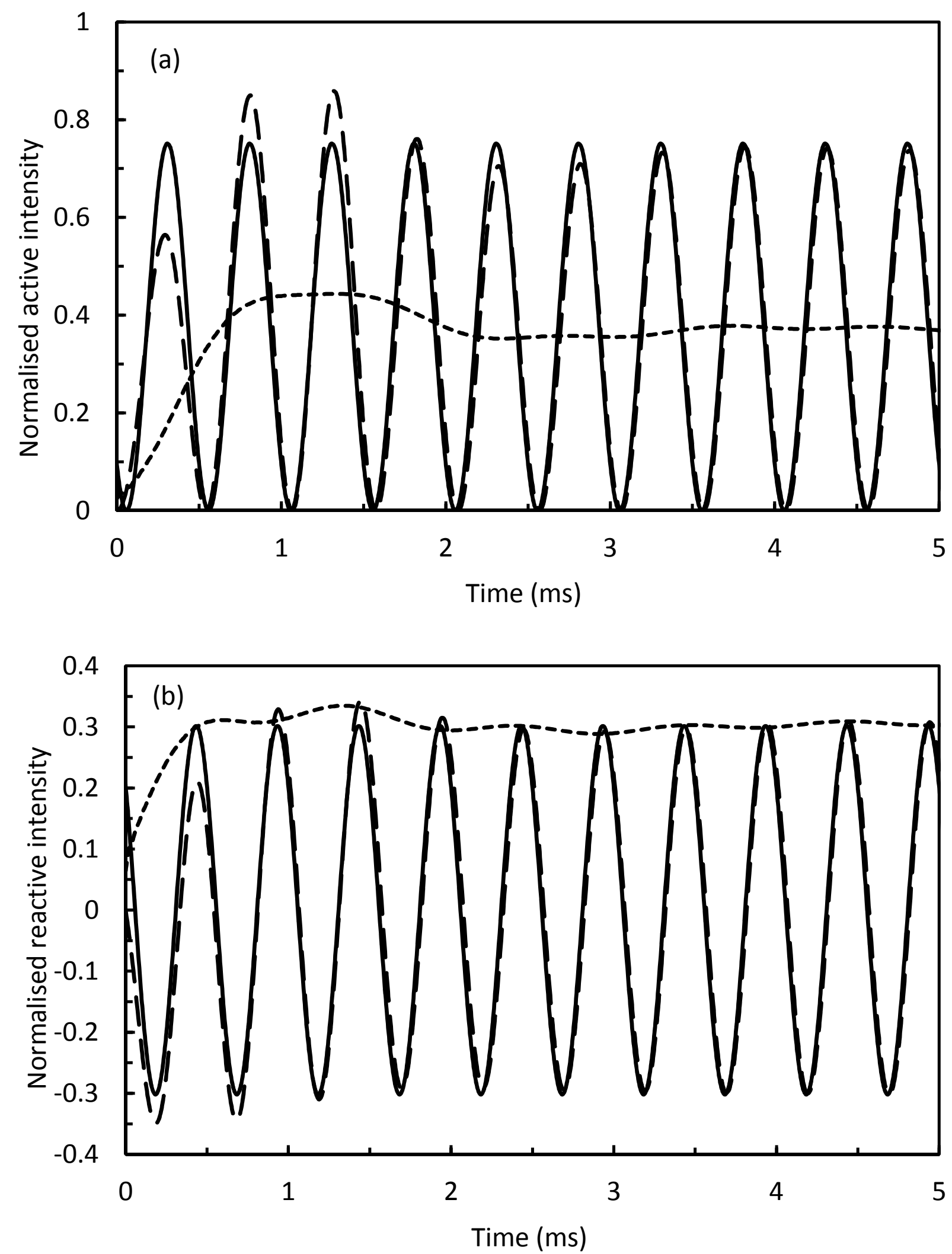

Fig. 7. Instantaneous complex intensity at $1000 \mathrm{~Hz}$ : instantaneous active (a) and instantaneous reactive $(\mathrm{b}):-\frac{}{\longrightarrow}$, theory; $-ー-$, experimental data based on method of Stanzial and Prodi ${ }^{10} ;$ - - - - -, experimental data based on method of Heyser ${ }^{7}$. 

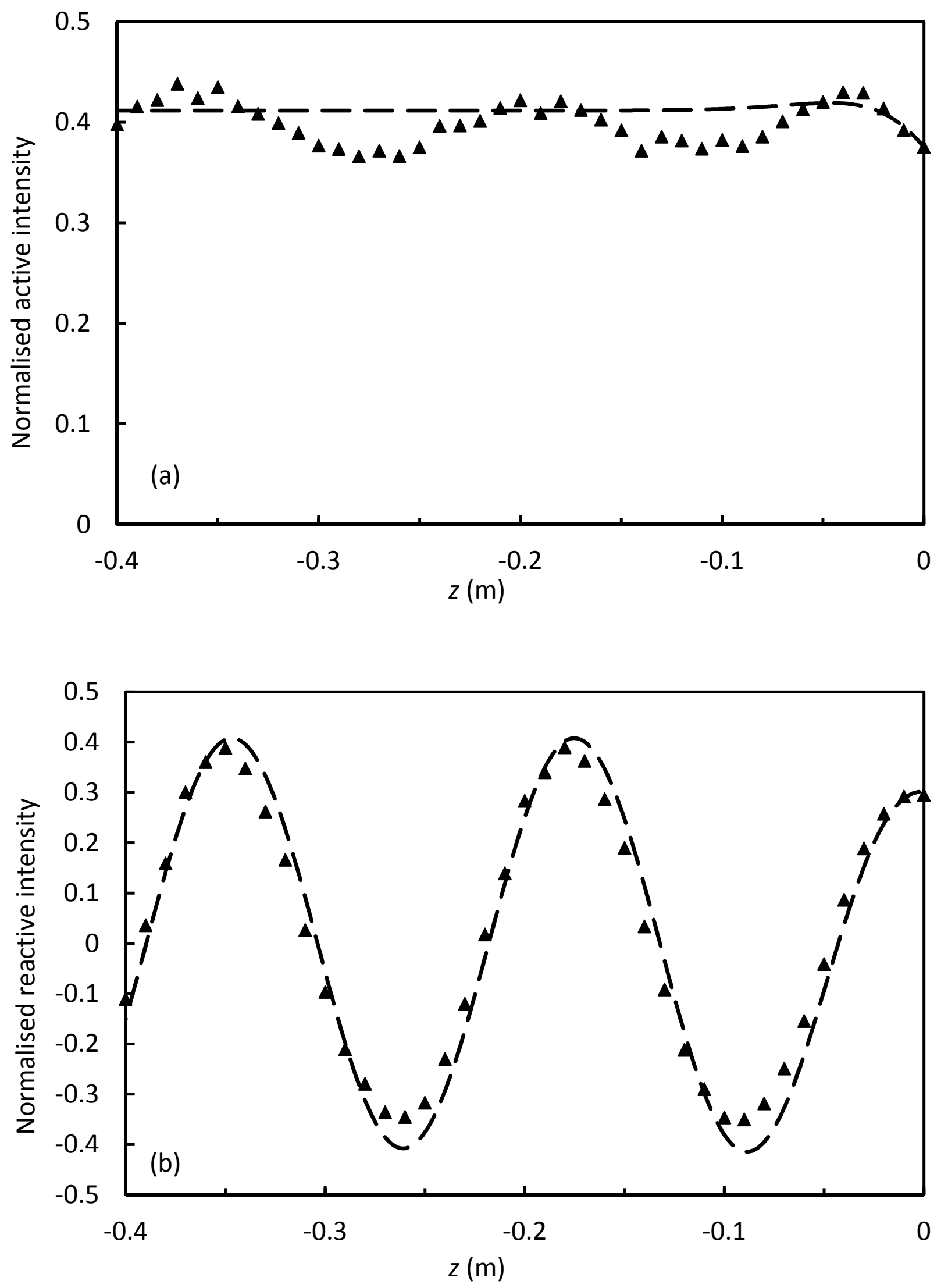

Fig. 8: Axial intensity at $1000 \mathrm{~Hz}$ : active (a) and reactive (b):

$\longrightarrow$, theory; $\boldsymbol{\Delta}$, experiment. 

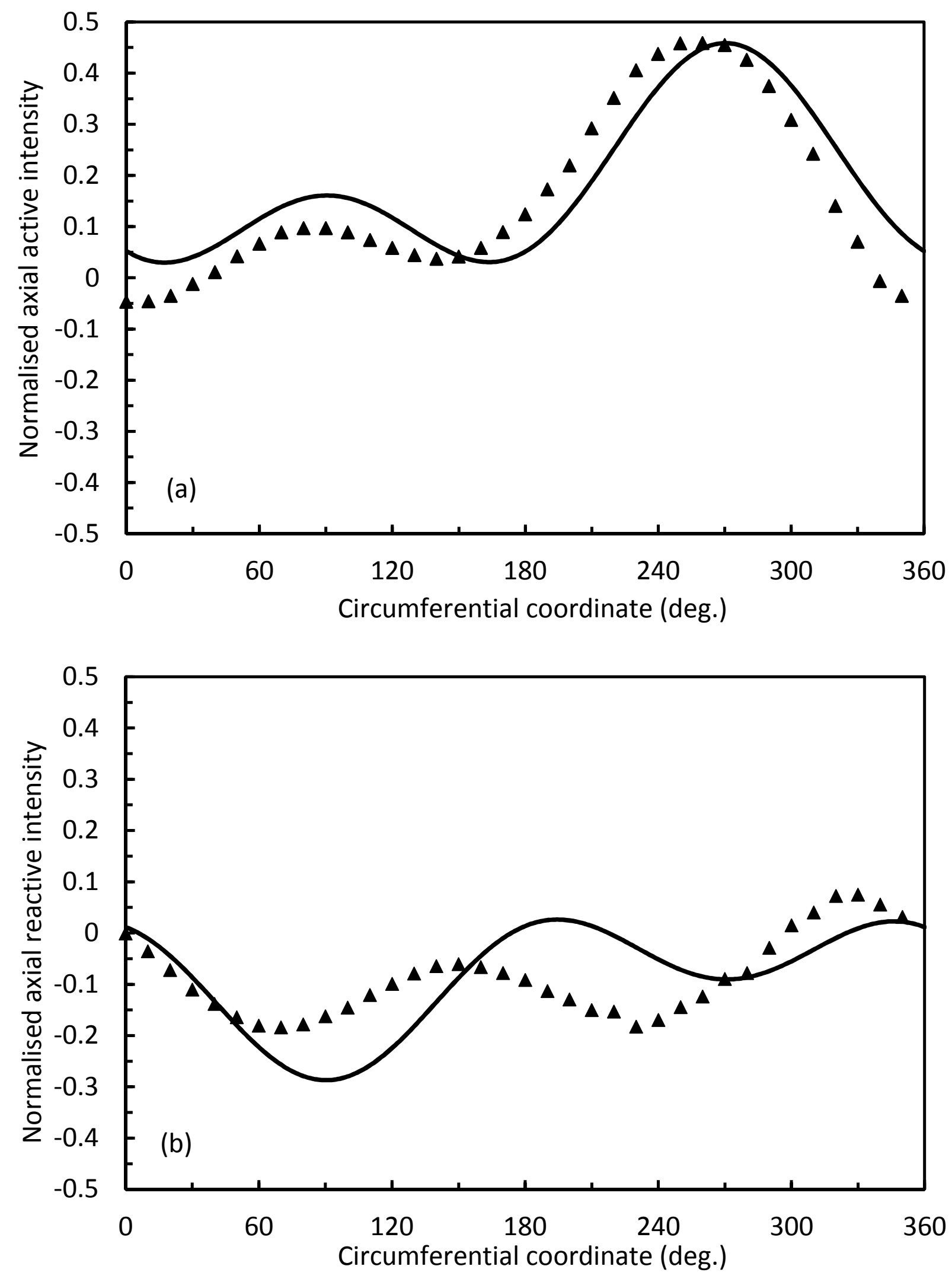

Fig. 9: Active (a) and reactive (b) axial intensity at 1800Hz:

$\longrightarrow$, theory; $\boldsymbol{\Delta}$, experiment 

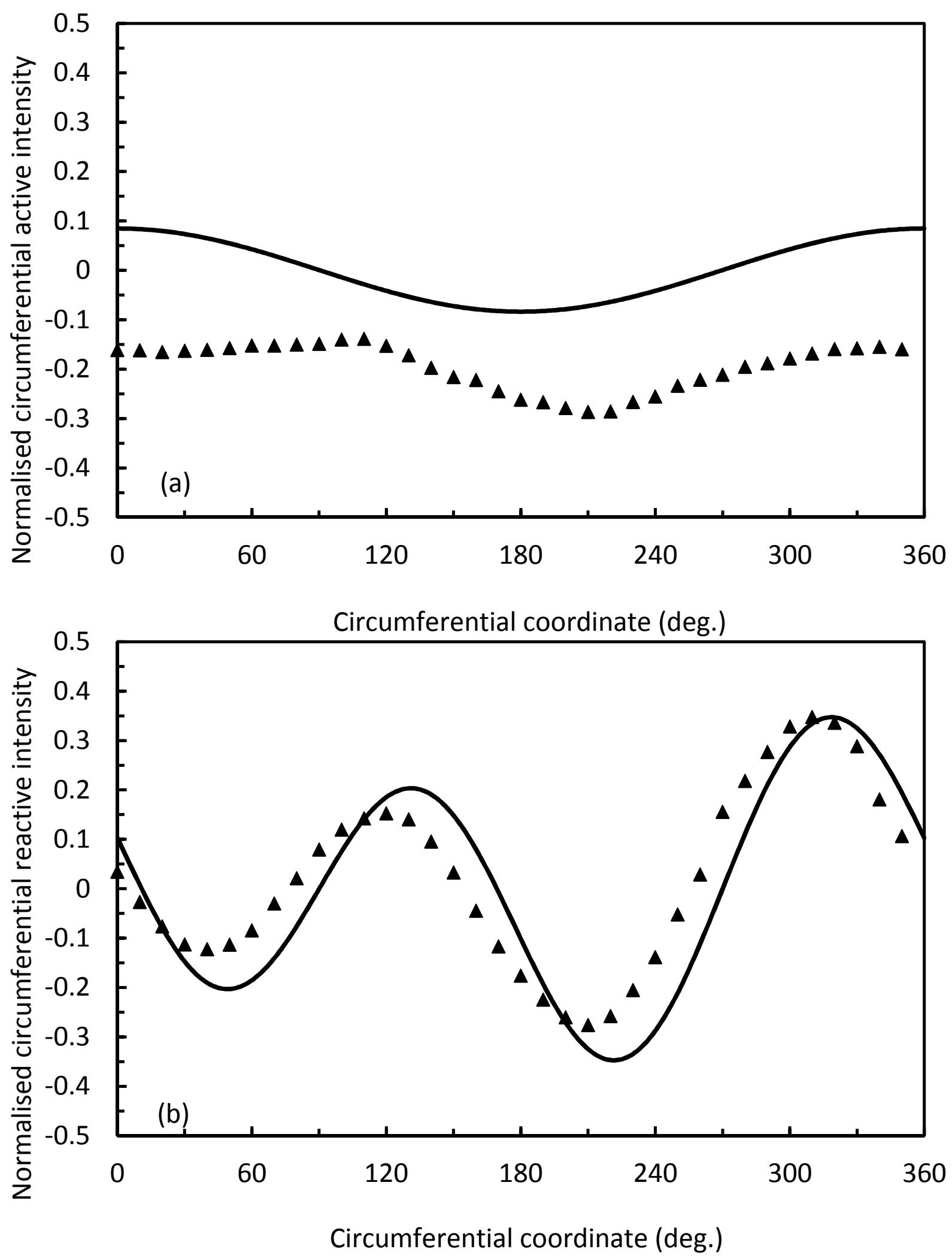

Fig. 10: Active (a) and reactive (b) circumferential intensity at 1800Hz:

$\longrightarrow$, theory; $\mathbf{\Lambda}$, experiment 

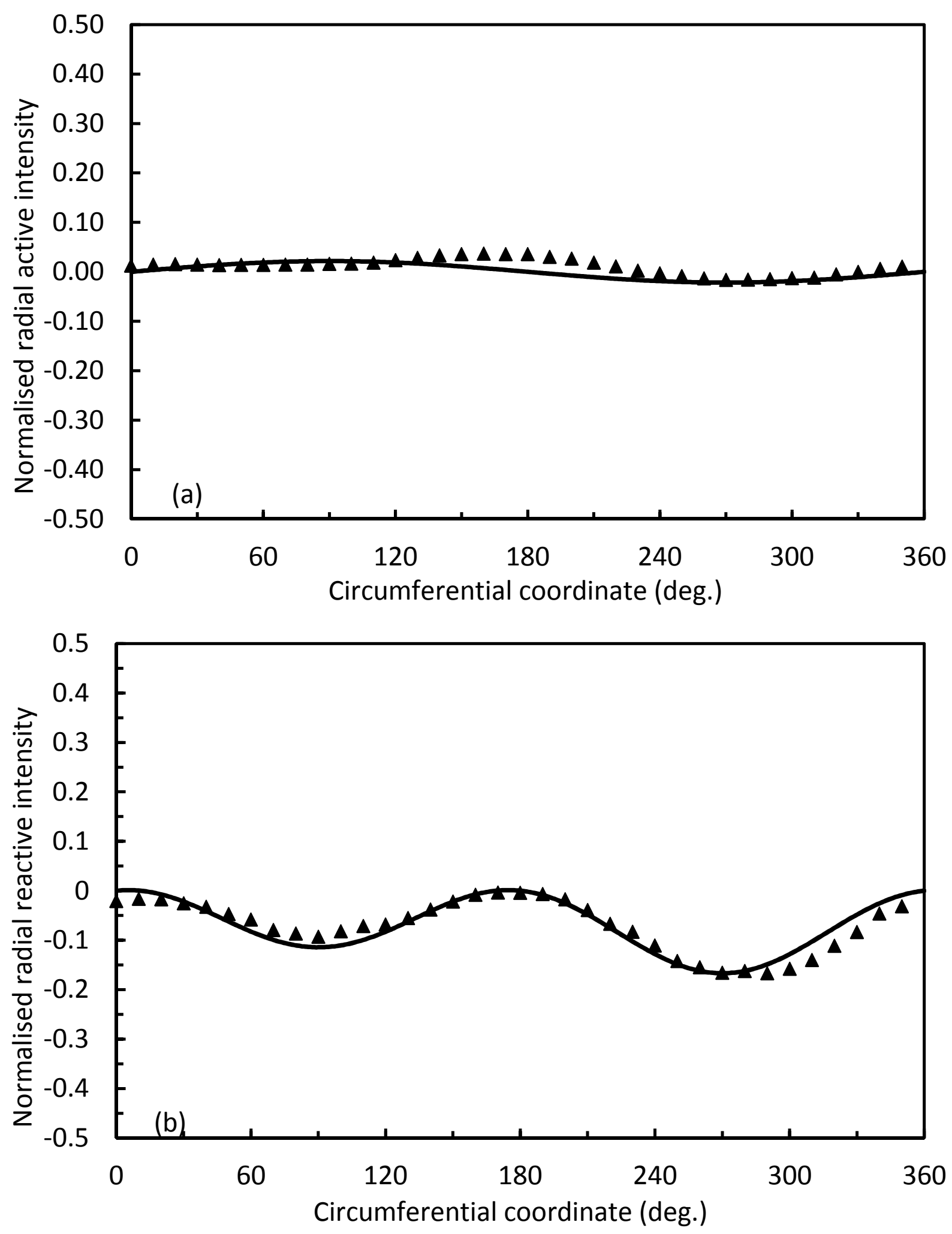

Fig. 11: Active (a) and reactive (b) radial intensity at 1800Hz:

$\longrightarrow$, theory; $\boldsymbol{\Lambda}$, experiment 